\title{
Understanding the Robot Ecosystem: Don't lose sight of either the trees or the forest
}

\author{
Dohoon Kim \\ Kyung Hee University \\ dyohaan@khu.ac.kr
}

\begin{abstract}
The robot sector in many countries has thrived recently thanks to government supports and innovations in various industries. This study, using the patent database to define the robot sector, reconfigures $I O$ (Input-Output) data to analyze the relationships among various sectors. In particular, we consider the internal description of the robot sector (mesoscopic view-the trees) as well as the relationship between the robot and the non-robot sectors (macroscopic view - the forest), so that we can not only understand robot ecosystems in various dimensions, but also develop policy insights. For the sake of systematic analysis of the intra- and inter-sector relations as well as the meso-macro links, this study constructs network models and employs several network measures. Our model and analysis present a good case study to understand the nature of the robot sector in terms of the business ecosystem. This novel approach also contributes to finding out a promising path that leverages the strengths of intrasector relations and spreads the impact of the robot sector across the macro relations.
\end{abstract}

\section{Introduction}

Technological progress propels economic growth and long-term industry changes. Despite burgeoning literature (e.g., [1], [11], and many), our understanding of how progress in one area is linked to other fields and spread throughout the economy is not perfect, particularly when new technology emerges (e.g., sharing economy platforms like Uber and Airbnb). Robots are also closely related to various economic activities, but our understanding of their impacts on and relationships with other areas is still lacking. Since robots, together with artificial intelligence and big data, are regarded as a key element in the Industry 4.0 and receive full supports from many governments, it is timely and necessary to conduct study for deeper understanding of robot's business ecosystem.

The potential of robots may be inherent in its innate nature as general purpose technology (GPT, [10], [26]). GPT is characterized by a catalyst for a broad range of technological improvement as well as an enabler of nationwide innovations. As GPT, robots are expected to promote knowledge creation and diffusion by establishing strong links between firms and their users and suppliers. Accordingly, the robot sector creates value by developing more efficient processes. ${ }^{1}$

Indeed, robotics and automation are dramatically reshaping the global economy and building its own business ecosystems around the world ([28]). With the proliferation of new production methods and innovations such as Industry 4.0, the demand for robots has increased significantly around the world in the last few years. Demand for industrial robots is expected to exceed at least 500,000 units by the end of 2018 ([23]), and this trend will lead to increased demand for service robots. Especially in Asian nations including China, Japan and South Korea, the demand for robots is overwhelming other regions (about $60 \%$ of the world's robots are populated in those countries, [9]). For example, Japan has six out of the top 10 (in terms of sales revenue) industrial robot manufacturers. In Korea, the number of robots per thousand workers is 60 , which is more than twice that of Japan and Germany (in this index, Korea ranks first in the world; the global average is less than 90). There is also a survey report demonstrating that robot industries are contributing around $3 \%$ of GDP growth in OECD countries ([9]). The current situations and trends suggest that, in the Industry 4.0 era, appropriate policies developed based on a more holistic and detailed understanding of the robot ecosystem will have a greater impact on the entire economy.

In these backgrounds, the purpose of this study is to develop a framework for analyzing the structural properties of a newly emerging techno-economic sector like the robot sector, thereby suggesting insights into an effective development path. To achieve this goal, we first examine the robot sector from multiple angles,

\footnotetext{
${ }^{1}$ Our research focuses on the industrial robots. Refer to Table 1 in section 4.1 for the definition of the robot sector
} 
which is expected to provide a novel way to derive the full potential of this sector. In particular, we analyze the various relationships related to the robot sector from the mesoscopic (the trees) as well as the macroscopic (the forest) perspectives. This approach will help to deeply understand robotic technologies and services based on the notion of the business ecosystem. In order to systematically analyze both internal (intra-sectoral) and external (inter-sectoral) relationships of the robot ecosystem, we employ network models, which are constructed with IO (Input-Output) data. Modelling and analyzing economic activities and business transactions based on the IO framework together with patent database complements the existing network measures that can only assess purely structural aspects, and enables rich analysis incorporating flows.

The organization of the paper is as follows. The next section briefly reviews literature on business ecosystem, IO framework and network analysis in relation to this research. Section 3 introduces our approach and framework based on IO data and network models together with some structural measures. Section 4 presents the analysis results from both the mesoscopic and macroscopic perspectives. The last sections discuss our findings and conclude the paper.

\section{Literatures and research backgrounds}

[20] argued that economic structure is of first-order importance to understand cross-country income differences. The economic structure, however, has been greatly affected by technological development and innovations triggered by GPT. The Industry 4.0 leverages multiple technologies such as AI, big data, IoT, and robots, which eventually transform the entire production method. Accordingly, it is highly regarded as a breakthrough innovative scheme led by GPT (for this reason, it is also referred to as the Fourth Industrial Revolution). Since these changes will have large and lasting impacts on the whole society and economy, many countries are paying attention to the Industry 4.0. In this context, a robust ecosystem built around robots serves as an essential conduit for a broad and extensive innovation progress.

In a similar vein to the notion of economic structure highlighted by Hirschman, the concept of 'business ecosystem,' which emphasizes the architectural aspects of economic relations, can be better applied to today's Industry 4.0 economy. [21], [22]. and [30] suggested employing ecological concepts and approaches to business research in order to complement the existing research framework that does not catch up with the changing business and technology environment. With the spread of IT and digital convergence, industrial boundaries have collapsed and it is necessary to escape from the framework of adhering to industrial silos. The ecosystem framework has focused on the interactions among various sectors and resulting synergies. Thanks to the pioneering studies, the notion and framework of business ecosystem has been widely adopted in many fields: to name a few, [5], [6], [34] for mobile communications and [2], [3], [4], [24] for computer software.

The results of the prior studies extended the existing popular paradigm of the-structure-lead-the-performance in industrial economics and enabled analysis of structural characteristics of various inter-sector interactions and their performance. For example, the pre-existing network structure in patent database has strong predictive power on future innovation ([1]). The ecosystem analysis also incorporate technology- or sector-specific data such as M\&A and collaboration data for identifying keystones in an ecosystem: e.g., mobile ecosystem ([5], [6]). Finally, ecosystem studies have come to address a nation-wide economy or global value chain from a macroscopic perspective ([21]).

In the course of such development, this study takes a somewhat different perspective on the notion of the business ecosystem. In other words, while the traditional notions as in [22], [30], etc. usually address the relationships at the firm level, the ecosystem in this study deals with the structural relations at the industry level. In fact, this perspective and approach are not new. Some prior studies like [34], as well as the industrial ecology studies, usually consider the ecosystems at the industry level.

The advancement and proliferation of the ecosystem studies has developed more systematic methodologies that were limited to qualitative analysis and case studies in the early stage. For example, the development presented many ways to operationalize and quantify key notions (e.g., keystones and niches) and conceptual measures for ecosystem healthiness including productivity (e.g., the degree of resource or material outflows to inflows), spillover (e.g., the total impact on the whole ecosystem), and coverage (e.g., affecting and affected entities) ([22], [30], [31]). Furthermore, many prior works have incorporated network models to this area, opening a new window of utilizing rich theories and concepts developed in the social network studies. For example, incorporating the notion of structural hole ([12]) makes it possible to identify entities that serve as bridges in ecosystems or to quantify resilience. Other network measures help to explore symbiotic relations for a more constructive understanding of the ecosystems. As a matter of fact, we believe that the network theory presents the best methodological frame for describing and analyzing the business or industrial ecosystems. Indeed, many ecosystem studies represent their ecosystems with various networks (e.g., [4], [5], [6], [7], 
[24], [34], [35]). The network approach is also flexible enough to represent and deal with different levels of ecosystems (e.g., at the firm or industry level) in a consistent way.

In the background and context above, this study considers robots, which has few prior studies from the ecosystem point of view. Although we observe new phenomena related to robots (e.g., robots with AI, robots for smart homes, etc.), we focus on the industrial robots. Thus, this sector is not in the nascent stage of its lifecycle and is still evolving.

While many ecosystem studies take the microscopic perspective at the firm level, this study addresses robots from both meso- and macroscopic perspectives at industry or sectoral level. Based on this approach, we try to explain the overall structure of the robot-related interactions and obtain a holistic view of their relations through analyzing them from multiple angles. Further, we construct network models with IO data to analyze the flow characteristics as well as the structural aspects of the interactions. IO model and analysis are particularly well suited with the ecosystem frame since IO data is able to show a detailed structure and interactions in the economy ([16], [17], [29], [33]). Therefore, employing IO model helps us to develop useful measures (e.g., spillover effects), in addition to quantitatively exploring structural properties. Through this approach, we examine the robot areas (largely unexplored and unexplained despite being in full operation) and address the new ecological role of the robot sector.

\section{Methodology and models}

\subsection{IO model}

The IO (Input-Output or inter-industry) framework, developed by Leontief ([27]), describes a complete picture of the flows of goods and services in an economy for a given year. In particular, the numbers in the main table (see matrix $\mathbf{X}$, called 'intermediate demands') represent the interdependencies among various sectors. Thanks to its neat format and structural properties together with global standardization efforts (e.g., World IO Database, [36]) the IO model presents an effective frame to document sectoral interdependencies. Thus, it has been popular and proven useful in diverse contexts and application areas (e.g., [8], [13], [15], [19], [25]). For example, how differences in business characteristics across industries - as captured by IO linkages between sectors-affect cross-industrial gaps in outputs, valueadded, employment, etc.

$$
\mathbf{x}=\mathbf{X} \mathbf{1}+\mathbf{d}
$$

, where $\mathbf{X}=\left[X_{i j}\right]_{i, j=1 \cdots n}, \quad \mathbf{1}=[1 \cdots 1]^{T}, \mathbf{x}=\left[X_{1} \cdots X_{n}\right]^{T}$ and $\mathbf{d}=\left[\begin{array}{lll}d_{1} & \cdots & d_{n}\end{array}\right]^{T}$. The sizes of column vectors are all $\mathrm{n}$, the number of sectors. $X_{i j}$ captures the material flow or business transaction between sector $\mathrm{i}$ and $\mathrm{j}$ (i.e., supply from $\mathrm{i}$ to $\mathrm{j}$ ). $\mathbf{x}$ and $\mathbf{d}$ represent total outputs and final demands, respectively.

Each column $\mathrm{j}$ of $\mathbf{X}\left(\left[\begin{array}{lll}X_{1 j} & \cdots & X_{n j}\end{array}\right]^{T}\right)$ represents a producing sector, where the corresponding row elements record the amount of inputs to $\mathrm{j}$. Hence, each row $i$ of $\mathbf{X}$ enumerates sector i's output used as inputs to various sectors from 1 to $\mathrm{n}$.

We now define technology (or input) coefficients $a_{i j}$ 's: that is, $a_{i j}=X_{i j} / X_{j} . a_{i j}$ represents cents of $i$ 's output (in row $i$ ) used per dollar output of $j$ (in column j). Then, equation (1) can be transformed as follows.

$$
\mathbf{x}=\mathbf{A} \mathbf{x}+\mathbf{d} \quad \text { or } \quad(\mathbf{I}-\mathbf{A}) \mathbf{x}=\mathbf{d}
$$

, where $\mathbf{A}=\left[a_{i j}\right]_{i, j=1 \cdots n}$ and $\mathbf{I}$ is an identity matrix.

The matrix $(\mathbf{I}-\mathbf{A})$ is called Leontief matrix, whose inverse has special meaning. Since $\mathbf{I}+\mathbf{A}+$ $\mathbf{A}^{2}+\cdots=(\mathbf{I}-\mathbf{A})^{-1}$, this inverse summarizes all the intermediate effects and quantifies the intensity of causation among sectors. Thus, the row [column] sums of $(\mathbf{I}-\mathbf{A})^{-1}$ represent the production-induced [inducing] effect of corresponding sectors based on the technology coefficient matrix $\mathbf{A}$ (called multiplier or spill-over effect in [18], [35]).

[33] first developed more sophisticated metrics based on the multipliers and presented the notions of effect and response ratios. The effect ratio of sector $i$ is the gap between i's production-inducing effect and the overall average. The response ratio is similarly defined. Thus, the sector whose effect [response] ratio is bigger than 1 is relatively more effective in [sensitive to] productions of other sectors.

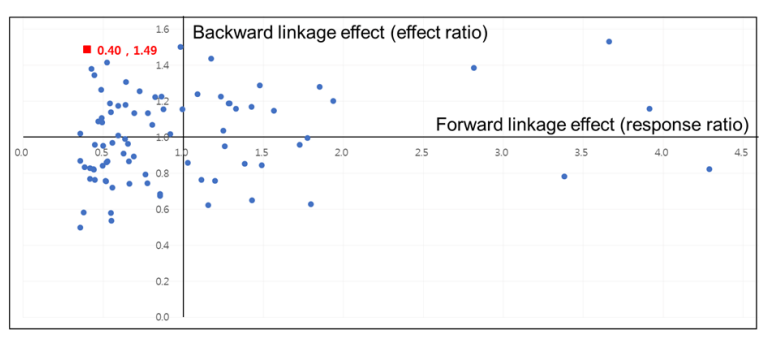

Figure 1. IO analysis: back- \& forward links

Figure 1 depicts the effect and response ratios (or, backward and forward linkage effects resp.) of all the sectors. Here, the original 2016 IO table was modified and reconstructed by adding a new sector (the robot sector) and (accordingly) rearranging the entire flows (refer to our sector definition and intra-sector table- 
Table 1 and 2-in Section 4.1). The robot sector shows high (above average) backward effect and low (below average) forward effect, which means this sector belongs to the production resource category (e.g., general machinery and automotive) in the macroscopic perspective.

\subsection{Network ecosystem model with IO data}

Typical measures in the IO framework include productivity (the ratio of resource or material outflows to inflows), spillover (the chain effect on the whole ecosystem), and coverage (the size of affecting or affected sectors). Moore ([30], [31]) and Iansiti ([21], [22]) also consider similar measures for exploring symbiotic relations and identifying hubs or keystone sectors. This study employs the relevant metrics to examine and evaluate the robot sector in the mesoscopic perspective (intra-sector relations) as well as the macroscopic perspective (inter-sector relations). Since the ecological approach is more effective when applied to network structures, the measures are evaluated on the network structure derived from the intra- and intersector IO matrices. We also employ other networkoriented measures for a systematic and integrated understanding of the robotics sector.

First, ignoring $\mathbf{d}$ in (1) and $(2),{ }^{2}$ we re-define the following notations: $X_{j}=\sum_{i} X_{i j}$ and $X^{i}=\sum_{j} X_{i j}$, which denote the total input into sector $\mathrm{j}$ and the total output from sector $\mathrm{i}$, respectively. Incorporating full IO data (without introducing network structure), the traditional (demand-driven) Leontief technology coefficients (e.g., Table 2) and (supply-driven) GhoshGruver production coefficients are defined as follows:

$$
a_{i j}=X_{i j} / X_{j} \text { and } r_{i j}=X_{i j} / X^{i} \text {. }
$$

With these matrices, the input and output coefficients of sector $i$ are attained by summing $a_{i j}$ 's and $r_{i j}$ 's in the respective row and column. That is,

$$
a_{i}=\sum_{j} a_{i j} \text { and } r_{i}=\sum_{j} r_{j i} \text {. }
$$

The productivity of sector $\mathrm{k}$ is then defined as follows:

$$
\xi_{k}=r_{k} / a_{k} \text {. }
$$

This is the ratio between the input and output coeff., which measures the rate of value creation at the sector.

For the sake of closer examinations of structure characteristics of the symbiotic relationship among sectors, we construct networks on the basis of IO data

\footnotetext{
2 This is to focus on sectoral inter-relations. In this sense, our approach is somehow different from the typical IO analysis in economics, and it is rather closer to utilizing the IO framework in ecological studies.
}

and apply the following network measures. First, the intra- and inter-sector IO networks are derived from the respective IO matrices. In particular, the size of the multiplier effect captured in the technology coefficient matrix $\left(a_{i j}\right)$ is used as the basis for establishing a directional relationship (i.e., arcs $(i, j))$ between the sectors. $^{3}$ Setting the threshold at 0.01 , we build two networks, each of which represents the macroscopic structure of inter-sector relations in the entire economy ('macro network' for short) and the mesoscopic structure inside the intra-robot sector ('meso network'), respectively.

Upon the networks constructed as above, the following network measures are applied to discover and assess their ecological characteristics. We first calculate traditional network metrics of degree centralities both on directed ( $\alpha^{\text {in }}$ and $\alpha^{\text {out }}$ ) and on underlying undirected networks $(\alpha)$. Other classical metrics such as the betweenness centrality $(\beta)$ and the closeness centrality $(\gamma)$, and the clustering coefficient $(\theta)$ are also worked out.

Moreover, we employ the notion of structural hole ([12]), which represents the relationship of nonredundancy. If a sector is directly connected to some sectors which simultaneously support transactions with each other, then the inter-dependency among these sectors will be high. On the other hand, so-called 'bridge' connects diverse sectors and facilitates access and exchange of information and knowledge. Following [12] and [32] (and many other prior studies), we assume that innovations and entrepreneurial opportunities arise from the structural holes. Our approach to the structural hole employs two traditional indices-efficiency and constraint-, customized to incorporate IO data.

The efficiency of sector $\mathrm{i}$ measures the relative importance of diversity in the network based on the network size $\left(N_{i}\right)$ and the level of non-redundancy $\left(T_{i}\right)$. Our metric also utilizes transaction data (i.e., arc flows as weights) as well as network topology in quantifying the non-redundancy levels, which is different from typical approach solely relying on unweighted graph. For this purpose, we define the following two terms:

$$
\rho_{i q}=\frac{X_{i q}+X_{q i}}{X_{i}+X^{i}} \text { and } \tau_{i q}=\frac{X_{i q}+X_{q i}}{\max _{k}\left(X_{i k}+X_{k i}\right)}
$$

where $\rho_{i q}$ and $\tau_{i q}$ represent the importance of direct link between $\mathrm{i}$ and $\mathrm{q}$ (or the relative strength of the tie) and the marginal intensity of sector $\mathrm{i}$ in relation with $\mathrm{q}$, respectively. That is, $\rho_{i q}$ means the proportion of direct $\mathrm{i}-\mathrm{q}$ transaction relative to total economic relations

\footnotetext{
${ }^{3}$ Since this multiplier effect depends to a large extent on the number of sectors to which a given sector is connected (cf. outdegree) and the intensity (cf. input coefficient) with which its output is used as an input by the other sectors, some prior studies ([14], [35]) built networks upon the technology coefficient matrix.
} 
established by $\mathrm{i}$, whereas $\tau_{i q}$ means the relative strength of $\mathrm{i}-\mathrm{q}$ relation to the biggest one that sector $\mathrm{i}$ holds. Following [12], the efficiency factor is then defined as follows:

$$
\epsilon_{i}=T_{i} / N_{i}
$$

where $T_{i}=N_{i}-\sum_{j} R_{i j}$ and $R_{i j}=\sum_{q} \rho_{i q} \tau_{j q}$. Here, $R_{i j}$ represents the level of redundancy in $\mathrm{i}-\mathrm{j}$ relation and $T_{i}$ the degree of non-redundancy of sector i. That is, $T_{i}$ captures the effective degree of sector i through nonredundant connections. $\epsilon_{i}$, the normalization of $T_{i}$, represents the diversity of sector $i$ in the network and takes values between 0 and 1 ( 1 for the maximum efficiency).

However, a high-efficiency sector may strongly depend on other sectors, which can limit the realization of efficiency. Therefore, [12] also suggested another metric called 'constraint index' to complement the efficiency index. The constraint index $\omega_{i}$ represents i's sectoral dependency and reflects i's efforts or investments to reach other contacts.

$$
\omega_{i}=\sum_{j} w_{i j},
$$

where $w_{i j}=\left(r_{i j}+\sum_{q} r_{i q} r_{q j}\right)^{2}$ with $r_{i j}$ defined above (i.e., the production coefficient, which is calculated with IO matrix on the corresponding network). The higher the average of $\omega_{i}$ 's, the fewer the structural holes in the overall network.

In sum, the structural indices measure local (at the level of (sub-)sector k) and global (in the entire network scale) characteristics of (sub-)sectoral interrelationships to figure out the role of the robot sector for our economy in the macro- as well as mesoscopic perspectives. The following indices are employed here for both macro and meso networks.

- $\operatorname{productivity~}\left(\xi_{k}\right)$

- degree centralities: in- and out-degree centralities $\left(\alpha_{i}{ }^{\text {in }}\right.$ and $\alpha_{i}{ }^{\text {out }}$ ) on di-graph as well as total degree centrality $\left(\alpha_{i}\right)$ on underlying undirected network

- betweenness centrality $\left(\beta_{i}\right)$

- closedness centrality $\left(\gamma_{i}\right)$

- clustering coefficient $\left(\theta_{i}\right)$

- efficiency factor $\left(\epsilon_{i}\right)$

- constraint factor $\left(\omega_{i}\right)$

\section{Data and analysis}

\footnotetext{
${ }^{4}$ We intentionally used the 2016 database (not the most recent one) in order to maintain consistency with the IO data. The most recent IO
}

\subsection{Data and IO reconfiguration}

The robot sector spans several sectors in the typical IO frame, including general machinery and computer software (the typical IO frame does not have a classification code for the robot sector). We first define the robot sector by selecting the most relevant sectors (in terms of the standard industry classification (SIC) that follows mostly the OECD classification system) based on the patent records. Although the SIC systems of the patent database and the IO database are generally different, the two systems in Korea are very similar. Moreover, the minor differences (not many of these cases) can be easily mapped. In short, when a mismatch occurred, we have further refined (i.e., one step down) or aggregated (i.e., one step up) the classifications of one database to match both. For example, 'Agriculture (code: A01A),' 'Livestock (A01B),' 'Forestry (A01C),' and 'Fishery (A01D)' in the patent database were aggregated into A01, which corresponds to 'Agriculture, Forestry, and Fishery (code: 01)' in the SIC system.

We searched 2016 Korean patent database and specified 17 sectors (in terms of SIC) ranging from plastic manufacturing to software and IT services (refer to Table 1 and 2). ${ }^{4}$ The selection criterion is the percentage of patents related with design, production, and application of robot. That is, a sector whose patents pertaining to these categories accounts for more than $10 \%$ of its total patents is assumed to fall into the robot sector.

Table 1. Robot sector definition

\begin{tabular}{|c|l|}
\hline Sector code & \multicolumn{1}{|c|}{ Description } \\
\hline 023 & Plastic manufacturing and products \\
\hline 024 & Rubber manufacturing and products \\
\hline 031 & Metal manufacturing and products \\
\hline 032 & General machinery \\
\hline 033 & Special purpose machinery \\
\hline 034 & Electrical equipment \\
\hline 035 & Semiconductor \\
\hline 036 & Electrical display and devices \\
\hline 037 & Miscellaneous electronic components \\
\hline 038 & Computers and peripherals \\
\hline 039 & $\begin{array}{l}\text { Communications, broadcasting, video \& sound } \\
\text { equipment }\end{array}$ \\
\hline 040 & Household electrical appliances \\
\hline 041 & Precision machinery and equipment \\
\hline 042 & Automobile \\
\hline 045 & Miscellaneous manufacturing \& toll processing \\
\hline 061 & IT services \\
\hline 062 & Computer software development \\
\hline
\end{tabular}

data was published by the Bank of Korea in 2017 (the official extension of 2015 IO data), which counted transactions in 2016. 
The original IO table is composed of 82 sectors (the intermediate level of classification). As the new sector (code 100 is assigned for the sake of easy recognition) is added, the entire original input-output flows need to be newly reconfigured. For doing this, we first assume that the data in the patent database accurately reflects the actual transactions across the entire economy. In other words, the proportion of robot-related patents in a sector is assumed to properly reflect the fraction of the robot-related output in its total output. ${ }^{5}$ Then, the ratio of patents for the industrial robots in all industry standard codes are used for the reconfiguration as follows: that is, the original input to the other sectors from a specific sector is subtracted by this ratio (note that the underlying structure of the IO table can be seen as a representation of a bipartite directed network). This arrangement is carried out row by row on the original IO table. Lastly, adding the subtractions of the respective sectors constructs the transactions from the robot sector to all other sectors, which eventually fills in the new row (index: 100) for the robot sector in the newly rearranged IO table. This accomplishes not only the macroscopic composition of the inter-sector relations but also the mesoscopic composition of the intra-sector relations. For example, Table 2 shows the mesoscopic technology coefficient matrix calculated from the input-output flows among the sectors that constitute the robot sector.

Table 2. Intra-robot sector flows: technology coefficients

\begin{tabular}{|c|c|c|c|c|c|c|c|c|c|c|c|c|c|c|c|c|c|}
\hline & 023 & 024 & 031 & 032 & 033 & 034 & 035 & 036 & 037 & 038 & 039 & 040 & 041 & 042 & 045 & 061 & 062 \\
\hline 023 & 0.000 & 0.000 & 0.005 & 0.016 & 0.019 & 0.034 & 0.000 & 0.000 & 0.000 & 0.023 & 0.010 & 0.075 & 0.109 & 0.023 & 0.038 & 0.003 & 0.001 \\
\hline 024 & 0.000 & 0.000 & 0.001 & 0.007 & 0.010 & 0.005 & 0.000 & 0.000 & 0.000 & 0.002 & 0.001 & 0.008 & 0.008 & 0.008 & 0.004 & 0.000 & 0.000 \\
\hline 031 & 0.001 & 0.010 & 0.084 & 0.059 & 0.124 & 0.029 & 0.002 & 0.004 & 0.022 & 0.014 & 0.010 & 0.064 & 0.082 & 0.033 & 0.056 & 0.006 & 0.002 \\
\hline 032 & 0.002 & 0.012 & 0.022 & 0.203 & 0.095 & 0.015 & 0.003 & 0.005 & 0.012 & 0.004 & 0.002 & 0.070 & 0.008 & 0.036 & 0.026 & 0.020 & 0.007 \\
\hline 033 & 0.941 & 0.969 & 0.802 & 0.565 & 0.510 & 0.409 & 0.889 & 0.115 & 0.557 & 0.008 & 0.075 & 0.098 & 0.019 & 0.232 & 0.478 & 0.000 & 0.006 \\
\hline 034 & 0.001 & 0.001 & 0.008 & 0.044 & 0.058 & 0.310 & 0.007 & 0.029 & 0.089 & 0.107 & 0.066 & 0.069 & 0.066 & 0.033 & 0.025 & 0.053 & 0.006 \\
\hline 035 & 0.000 & 0.000 & 0.000 & 0.002 & 0.008 & 0.014 & 0.000 & 0.000 & 0.000 & 0.141 & 0.069 & 0.021 & 0.060 & 0.003 & 0.002 & 0.008 & 0.009 \\
\hline 036 & 0.000 & 0.000 & 0.000 & 0.006 & 0.011 & 0.002 & 0.000 & 0.000 & 0.000 & 0.228 & 0.108 & 0.015 & 0.109 & 0.001 & 0.004 & 0.000 & 0.000 \\
\hline 037 & 0.000 & 0.000 & 0.000 & 0.004 & 0.011 & 0.015 & 0.000 & 0.000 & 0.000 & 0.077 & 0.028 & 0.023 & 0.076 & 0.002 & 0.003 & 0.025 & 0.006 \\
\hline 038 & 0.000 & 0.000 & 0.000 & 0.003 & 0.001 & 0.001 & 0.002 & 0.011 & 0.007 & 0.288 & 0.007 & 0.000 & 0.004 & 0.002 & 0.003 & 0.295 & 0.639 \\
\hline 039 & 0.000 & 0.000 & 0.001 & 0.001 & 0.003 & 0.001 & 0.001 & 0.005 & 0.020 & 0.030 & 0.336 & 0.006 & 0.013 & 0.010 & 0.005 & 0.077 & 0.010 \\
\hline 040 & 0.001 & 0.000 & 0.002 & 0.002 & 0.000 & 0.001 & 0.000 & 0.000 & 0.000 & 0.000 & 0.000 & 0.445 & 0.000 & 0.001 & 0.003 & 0.003 & 0.001 \\
\hline 041 & 0.046 & 0.004 & 0.049 & 0.066 & 0.069 & 0.137 & 0.083 & 0.763 & 0.252 & 0.016 & 0.254 & 0.090 & 0.396 & 0.120 & 0.099 & 0.147 & 0.043 \\
\hline 042 & 0.001 & 0.000 & 0.003 & 0.003 & 0.021 & 0.001 & 0.000 & 0.001 & 0.000 & 0.001 & 0.000 & 0.001 & 0.000 & 0.464 & 0.006 & 0.003 & 0.006 \\
\hline 045 & 0.006 & 0.004 & 0.018 & 0.017 & 0.058 & 0.026 & 0.010 & 0.025 & 0.036 & 0.023 & 0.018 & 0.014 & 0.045 & 0.028 & 0.230 & 0.008 & 0.004 \\
\hline 061 & 0.000 & 0.000 & 0.000 & 0.001 & 0.001 & 0.001 & 0.000 & 0.001 & 0.001 & 0.001 & 0.001 & 0.000 & 0.001 & 0.000 & 0.002 & 0.046 & 0.042 \\
\hline 062 & 0.002 & 0.000 & 0.001 & 0.001 & 0.002 & 0.001 & 0.002 & 0.039 & 0.004 & 0.037 & 0.016 & 0.001 & 0.003 & 0.003 & 0.016 & 0.307 & 0.219 \\
\hline
\end{tabular}

\subsection{Results}

In macro and meso networks, the vertices represent sectors and the directed arcs imply relatively strong technological relationships. Similar to the approach of [34] and [35], we also set the cutoff value to 0.01. Thus, a directed arc $(i, j)$ between sector $\mathrm{i}$ and $\mathrm{j}$ is established when the corresponding technology coefficient $\left(a_{i j}\right)$ is greater than this cut-off. The primary goal of constructing the network based on the IO data is to minimize the loss of information and to reveal the essential structure of the transaction flow throughout the ecosystem. To achieve this goal, we tried several cutoffs between 0.005 and 0.05 and found that 0.01 would be the most appropriate level of the cut-off. Around the

\footnotetext{
5 It is rather a strong assumption since innovation activity and production activity do not coincide. This gap will also vary by sector, depending on sector characteristics and developmental levels. This inconsistency is inevitable, however, since it is not possible to consistently obtain or process all the necessary information from a
}

cut-off of 0.01 (between 0.008 and 0.018 ), the amount of transaction loss was in between $5 \%$ and $10 \%$ (in the macro network) or less than $2 \%$ (in the meso network). Both macro and meso networks are depicted below.

single database. Nonetheless, linking the patent data and the IO data in this way seems to be a less defective method and could be accepted in the perspective of operationalization in social science. We acknowledge this is a limit of our study and leave this issue for our future work (refer to the concluding part). 


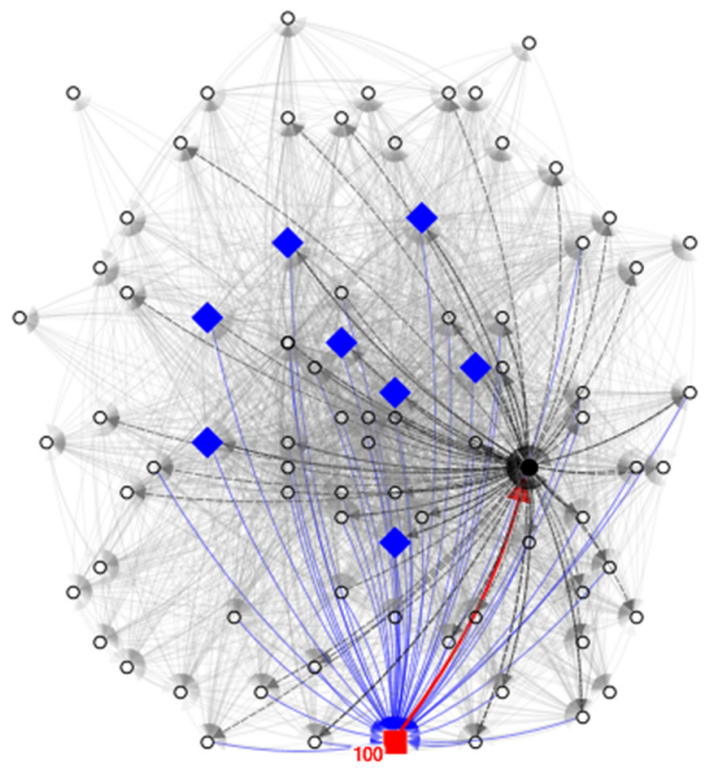

(a) macroscopic network structure

The solid square represents the robot sector. The blue arcs are directed to the sector and the red arc is going out from the sector. The diamonds are sectors whose zvalues of total degree $\left(\alpha_{i}\right)$ exceed 1.4 (no vertices with zvalue $>1.5$ )

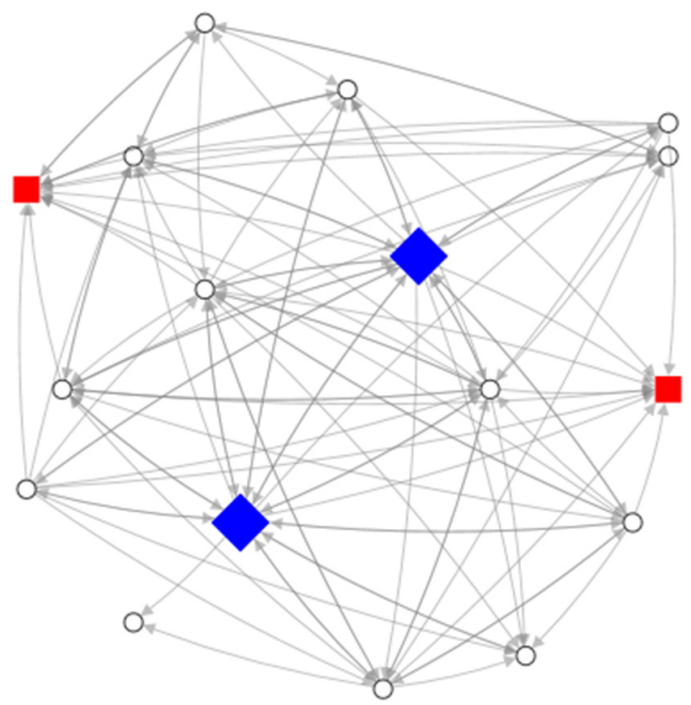

(b) mesoscopic network structure
The diamond represents the sectors -33 and $41-$ whose $z$-values of total degree $\left(\alpha_{i}\right)$ exceed 1.5. The squares are sectors -38 and $40-$ with high in-degree $\left(\alpha_{i}{ }^{i n}\right) z$-value (no vertices with $z$-value $>1.5$ ).

\section{Figure 2. Network structures (meso and macro)}

The robot sector in the macro network seems an absorber; that is, its in-degree surpasses the out-degree (only one outgoing arc to special purpose machinery (sector id: 33)). ${ }^{6}$ This observation is consistent with the nature of the robot sector shown in Figure 1. That is, the robot sector shows high effect ratio and low response ratio in typical spill-over analysis of the IO framework, which implies (relatively) high degree of backward linkage and low degree of forward linkage. Indeed, the robot sector in the inter-sector relations is not constrained, whose constraint factor is below the average.

The topological structure of the meso network shows quite different features from those of the macro network. The apparent differences can be confirmed by the differences in network indices (as summarized in Table 3 ). Although the size difference is one source of this difference (e.g., centralities), other measures that eliminate the scale effect (e.g., clustering coefficient) suggest that the meso network is flatter than the macro network. For example, while the average closeness centrality $^{7}$ of the meso network is higher than that of the macro network, the average clustering coefficients of meso is lower than that of macro. These differences imply that the sectors are more locally clustered (forming components) in the macro network than in the meso networks.

Based on the measures incorporating the flows on the network structure, the meso network (intra-sector structure) is more constrained than the macro network (inter-sector structure). Since both network show similar average efficiency, this difference may result in low average productivity of the robot sector (as a whole in the inter-sector relations). Indeed, the average productivity in the meso network (consisting of robotrelated sectors only) is lower than that in the macro network of the entire economy. Therefore, it can be concluded that the robot sector is not comparable to other highly productive sectors.

\footnotetext{
${ }^{6}$ In fact, 'special purpose machinery' is one of the core sectors that constitute the robot sector (Table 1). Despite subtracting the inputoutput flows associated with robots, the remaining part of this sector still has significant interaction with other sectors in the economy. This suggests that the relationship between the robot sector and the special purpose machines is very important.
}

\footnotetext{
7 Although this index is also affected by the network size, the scale effect is relatively smaller than the other centrality indices. However, the difference in this index between the two networks is significant considering the influence of the scale.
} 
Table 3. Structural indices (meso and macro)

\begin{tabular}{|l|l|l|}
\hline \multicolumn{1}{|c|}{ Measures } & \multicolumn{1}{|c|}{$\begin{array}{c}\text { Meso property } \\
\text { - mean (stdev) } \\
- \text { max \& min sectors }\end{array}$} & $\begin{array}{l}\text { Macro property } \\
\text { - robot sector (z-value) } \\
\text { - overall mean (stdev) }\end{array}$ \\
\hline Productivity & $-1.144(0.936)$ & $-0.927(-0.218)$ \\
& $-61(\max ) \& 37(\min )$ & $-1.801(4.003)$ \\
\hline Centrality & $-12.941(5.640)$ & $-50(-0.592)$ \\
(degree) & $-41(\max ) \& 24(\min )$ & $-69.590(33.111)$ \\
\hline Centrality & $-6.588(6.868)$ & $-3.282(-0.731)$ \\
(between) & $-62(\max ) \& 24(\min )$ & $-27.566(33.198)$ \\
\hline Centrality & $-0.045(0.007)$ & $-0.009(-0.420)$ \\
(closeness) & $-41(\max ) \& 24(\min )$ & $-0.009(0.002)$ \\
\hline Clustering & $-0.560(0.154)$ & $-0.715(0.626)$ \\
coefficient & $-62(\max ) \& 41(\min )$ & $-0.623(0.147)$ \\
\hline Efficiency & $-0.868(0.061)$ & $-0.812(-0.305)$ \\
factor & $-61(\max ) \& 62(\min )$ & $-0.840(0.089)$ \\
\hline Constraint & $-0.707(0.349)$ & $-0.000(-0.491)$ \\
factor & $-61(\max ) \& 24(\min )$ & $-0.303(0.617)$ \\
\hline
\end{tabular}

The indices regarding the meso network together with Figure 2-(b) suggest that machineries (sector id's 33 and 41) and IT areas (sector id's 61 and 62) play key role in the robot sector. On the other hand, the influence of the rubber sector (id: 24) is relatively weak. Pure structural indicators (e.g., degree indices) point out that the hubs are located at the machinery-related sectors. However, the IT service sector (id: 61) is ranked first not only in productivity $\left(\xi_{61}=4.206\right)$ but also in constraints factor $\left(\omega_{61}=1.241\right)$. Moreover, the computer software sector (id: 62) shows the highest clustering coefficient $\left(\theta_{62}=0.567\right)$ and betweenness centrality $\left(\beta_{62}=0.686\right)$. These findings imply that the IT area is leading the robot sector as another keystone in the intra-sector relations.

Structural hole analysis of the macro network (Figure 3-(a)) presents that the robot sector does not work as a broker in inter-sector relations. It is moderately efficient (slightly lower than the average) but not constrained. Thus, the robot sector seems loosely coupled with the other sectors in the entire economy.

From a mesoscopic point of view, the sectors involved are more cohesively connected with each other. Compared to macro networks, efficiency and constraint factors are both narrowly dispersed. Two sectorsrubber (id: 24) and house electronics (id: 40)-seem close to a broker. However, their influence (particularly, in terms of productivity and centrality) is not powerful enough to be called a broker.

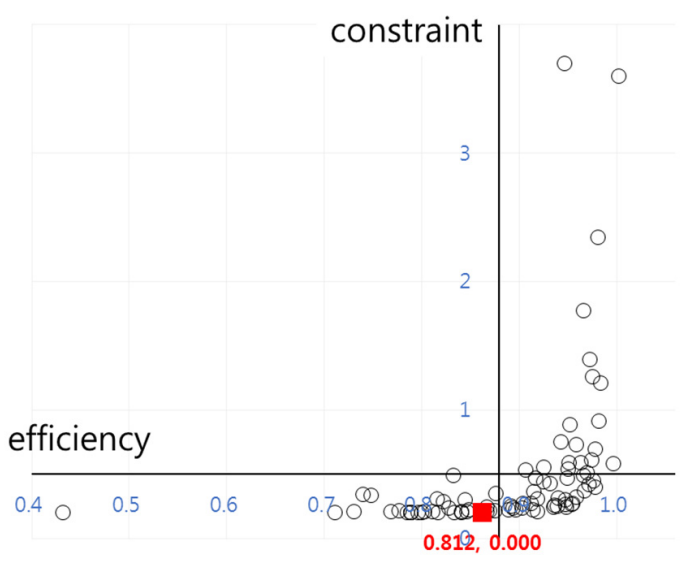

(a) macroscopic structural holes

Horizontal and vertical lines are drawn to intersect at the mean of the opposite axis. The red square indicates the robot sector with its efficiency and constraint coeff.

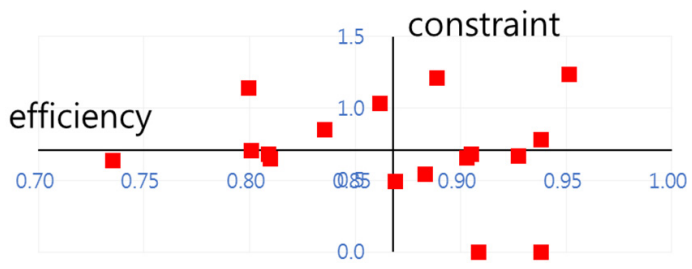

(b) mesoscopic structural holes

Horizontal and vertical lines are drawn to intersect at the mean of the opposite axis. Note the ranges of two axes: the efficiency factors are more densely populated (compare the standard deviations in Table 2).

Figure 3. Structural hole (meso and macro)

\section{Discussions}

This study demonstrates and analyzes the robot sector from both macro- and mesoscopic perspectives. This approach enhances our understanding of the robot sector to a multi-dimensional level by integrating the internal description of this sector with the external relations with other sectors in the entire economy. For example, our comparisons of the structural properties of the two networks suggest that while fairly cohesive intra-sector relations (inside the robot sector) do not allow room for structural holes, the robot sector itself (as a whole) plays a role of independent absorber in the inter-sector relations. We also found that the machinery sectors including general and special machineries (id: 32, 33 ) are keystones in the intra-sector as well as the intersector relations in Korea economy. The IT-related sectors such as communications, IT services, computer 
softwares (id: 39, 61, 62) play a similar role (albeit somewhat less impressive in terms of indices). As a result, we could propose a promising path from cohesive intra-sector relations led by machinery and IT to other machineries, IT services and electronics across the economy.

The proposed approach can also identify weakest links in the meso-macro connections. In the case of Korea, the weakest link lies at meager and inefficient forward linkage from the robot sector to the other sectors. To better understand this point, we examine the relationship between the structural measures and the performance (total output here) ${ }^{8}$ The following figures depict plots between the most influential measures and the economic outputs in the robot sector and the nonrobot sectors, respectively.

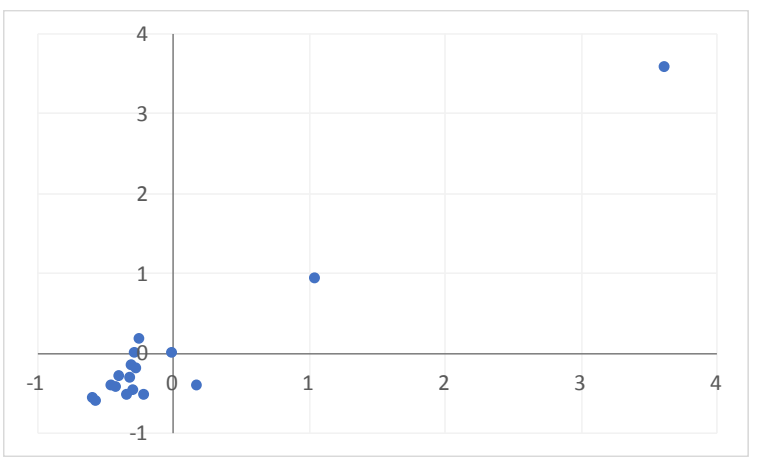

(a) robot sector: input coefficient vs output (sector) The plot between input coefficient and sectoral output (all in z-score). Although the size of data is not big enough to get meaningful regression, the input coeff. seems the most powerful explanatory variable.

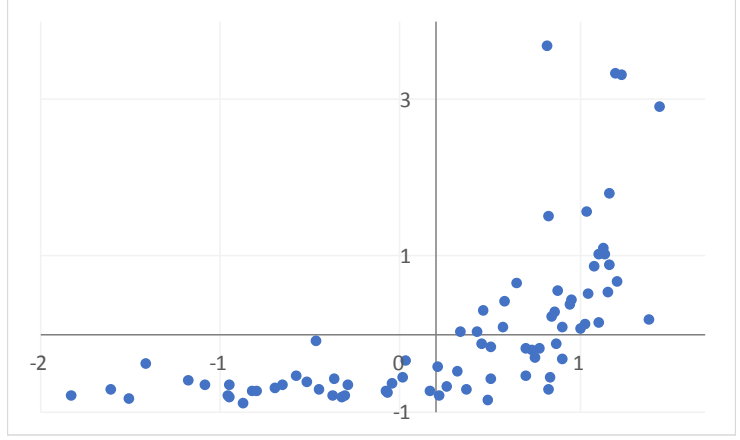

(b) non-robot sector: efficiency vs output (total)

The plot between efficiency and total output (all in zscore). The most significant explanatory variable in regression (with all normalized values) is efficiency.

\footnotetext{
${ }^{8}$ Employing output as a key performance indicator is common in the IO regime. This is to make the most of IO data
}

\section{Figure 4. Structure vs output}

Figure 4 also confirms this finding. The key dimensions that have the greatest impact on economic outcome or performance are different in the robot and non-robot sectors. The sectoral outputs in the robot sector have the largest positive correlation with the input coefficient measure, while the most powerful explanatory variable of multiple regression on the total output of the non-robot sector is the efficiency coeff., which accounts for structural hole. This also suggests that the proposed development path is highly likely to be implemented successfully. Note that in the success scenario above, leveraging highly cohesive intra-sector relations, keystone sectors (with high input coefficients) such as machinery and IT lead the entire robot sector while maximizing the ripple effect onto the whole economy through the linkage to the other sectors (with high efficiency factors).

Unfortunately, however, our data and analysis find out that the current inter-sector relations in Korea are not fully on the right track. For example, the robot sector itself in the macro network demonstrates weak efficiency and low degree of forward linkage. Moreover, as we have seen, our framework also helps to develop future policy directions to increase the chances of successful deployment of GPT like robots.

\section{Conclusion}

The robot sector in Korea has thrived recently thanks to government supports and innovations in various industries. Therefore, our analysis of economic activities and business transactions based on IO data together with patent database presents a good case study to understand the nature of the robot sector from the viewpoint of business ecosystem. For example, our approach to analyzing the robot sector by seeing the forest and the trees simultaneously, contributes to finding a promising development path that leverages the strengths of intra-sector relations and spreads the impact of the robot sector across the macro network. Our future work will focus on elaborating our idea of the mesomacro link and developing proper measures for integrated analysis of the two networks.

\section{References}

[1] Acemoglu, D., Akcigit, U., and Kerr, W.R. (2016). Innovation network. Proceedings of the National Academy of Sciences, 113(41), 11483-11488. 
[2] Baldwin, C.Y., MacCormack, A., and Rusnak, J. (2014). Hidden structure: using network methods to map system architecture. Research Policy, 43(8), 1381-1397.

[3] Baldwin, C.Y. (2012). Organization design for business ecosystems. Journal of Organization Design, 1(1).

[4] Basole, R.C. (2016). Accelerating digital transformation: Visual insights from the API ecosystem. IT Professional, 18(6), 20-25.

[5] Basole, R.C. (2009). Visualization of interfirm relations in a converging mobile ecosystem. Journal of Information Technology, 24(2), 144-159.

[6] Basole, R.C., Park, H., and Barnett, B.C. (2015). Coopetition and convergence in the ICT ecosystem. Telecommunications Policy, 39(7), 537-552.

[7] Basole, R.C., Russell, M.G., Huhtamäki, J., Rubens, N., Still, K., and Park, H. (2015). Understanding business ecosystem dynamics: a data-driven approach. ACM Transactions on Management Information Systems, 6(2), 6 . [8] Basu, S. (1995). Intermediate goods and business cycles: Implications for productivity and welfare. The American Economic Review, 512-531.

[9] BOK (2018). Status and tasks of global robot industry, International Economy Review, 2018-02, Bank of Korea, February 20, 2018.

[10]Bresnahan, T.F. and Trajtenberg, M. (1995). General purpose technologies engines of growth. Journal of econometrics, 65(1), 83-108.

[11]Brynjolfsson, E. and Hitt, L.M. (2000). Beyond computation: Information technology, organizational transformation and business performance. Journal of Economic perspectives, 14(4), 23-48.

[12]Burt, R.S. (1992). Structural Hole. Harvard Business School Press, Cambridge, MA.

[13]Ciccone, A. (2002). Input chains and industrialization. The Review of Economic Studies, 69(3), 565-587.

[14] Fadinger, H., Ghiglino, C., and Teteryatnikova, M. (2015). Productivity, networks and input-output structure: preliminary and incomplete. Annual Meeting of the Society for Economic Dynamics. Warsaw, Poland, 25-27.

[15]Finn, J.T. (1976). Measures of ecosystem structure and function derived from analysis of flows. Journal of Theoretical Biology, 56(2), 363-380.

[16] Ghosh, A. (1958). Input-output approach to an allocative system. Economica, 25, 58-64.

[17] Gruver, G.W. (1989). On the plausibility of the supplydriven input-output model: a theoretical basis for inputcoefficient change. Journal of Regional Science, 29, 441-450. [18] Han, S.Y., Yoo, S.H., and Kwak, S.J. (2004). The role of the four electric power sectors in the Korean national economy: an input-output analysis. Energy Policy, 32(13), 1531-1543.

[19] Hannon, B. (1973). The structure of ecosystems. Journal of Theoretical Biology, 41(3), 535-546.

[20] Hirschman, A.O. (1958). The strategy of economic development, No. 04; HD82, H5.

[21] Iansiti, M. and Lakhani, K.R. (2017). Managing our hub economy: strategy, ethics, and network competition in the age of digital superpowers. Harvard Business Review, 95(5), 8492.

[22] Iansiti, M. and Levien, R. (2004). Strategy as ecology. Harvard Business Review, 82(3).
[23]IFR (2017). World Robotics 2017, International Federation of Robotics (https://ifr.org/worldrobotics/)

[24]Iyer, B.R. and Basole, R.C. (2016). Visualization to understand ecosystems. Communications of the ACM, 59(11), 27-30.

[25] Jones, C.I. (2011). Misallocation, economic growth, and input-output economics. National Bureau of Economic Research, No. w16742.

[26] Kretschmer, T. (2012). Information and communication technologies and productivity growth.

[27]Leontief, W.W. (1936). Quantitative input and output relations in the economic systems of the United States. The Review of Economic Statistics, 105-125.

[28] McKinsey (2018). Robotics and Automation, McKinsey Global Institute (https://www.mckinsey.com/).

[29] Miller, R.E. and Blair, P.D. (2009). Input-Output Analysis, Cambridge University Press.

[30] Moore, J.F. (1993). Predators and prey: a new ecology of competition. Harvard Business Review, 71(3), 75-86.

[31] Moore, J.F. (2006). Business ecosystems and the view from the firm. The Antitrust Bulletin, 51(1), 31-75.

[32] Powell, W.W. and Grodal, S. (2006). Innovation networks. Fagerberg, J., Mowery, D.C., and Nelson, R.R. (ed.), The Oxford Handbook of Innovation.

[33] Rasmssen, P.N. (1956) Studies in Inter-Sectoral Relations, Amsterdam: North-Holland.

[34] Suh, Y. and Kim, M.S. (2015). Dynamic change of manufacturing and service industries network in mobile ecosystems: the case of Korea. Telematics and Informatics, 32(4), 613-628.

[35] Suh, Y. and Lee, H. (2017). Developing ecological index for identifying roles of ICT industries in mobile ecosystems: the inter-industry analysis approach. Telematics and Informatics, 34(1), 425-437.

[36] Timmer, M.P., Dietzenbacher, E., Los, B., Stehrer, R., and Vries, G.J. (2015). An illustrated user guide to the world inputoutput database: the case of global automotive production. Review of International Economics, 23(3), 575-605. 